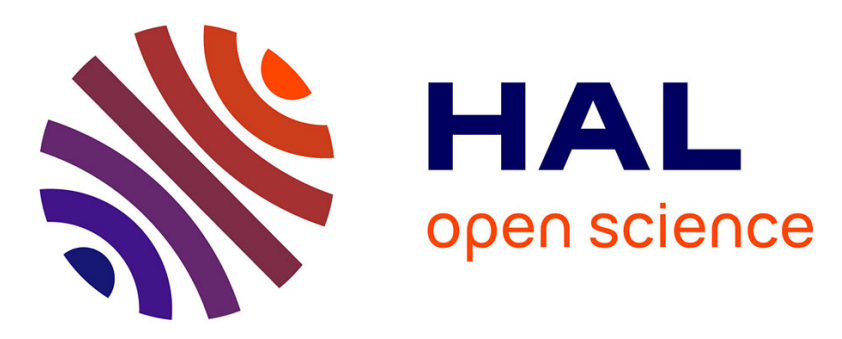

\title{
Computation of permeability with Fast Fourier Transform from 3-D digital images of porous microstructures
}

\author{
Hai Bang Ly, Vincent Monchiet, Daniel Grande
}

\section{- To cite this version:}

Hai Bang Ly, Vincent Monchiet, Daniel Grande. Computation of permeability with Fast Fourier Transform from 3-D digital images of porous microstructures. International Journal of Numerical Methods for Heat and Fluid Flow, 2016, 26 (5), pp.1328 - 1345. 10.1108/HFF-12-2014-0369 . hal01665962

\section{HAL Id: hal-01665962 \\ https://hal.science/hal-01665962}

Submitted on 28 Mar 2021

HAL is a multi-disciplinary open access archive for the deposit and dissemination of scientific research documents, whether they are published or not. The documents may come from teaching and research institutions in France or abroad, or from public or private research centers.
L'archive ouverte pluridisciplinaire HAL, est destinée au dépôt et à la diffusion de documents scientifiques de niveau recherche, publiés ou non, émanant des établissements d'enseignement et de recherche français ou étrangers, des laboratoires publics ou privés. 


\title{
Computation of permeability with Fast Fourier Transform from 3-D digital images of porous microstructures
}

\author{
Hai-Bang Ly ${ }^{\mathrm{a}, \mathrm{b}}$, Vincent Monchiet ${ }^{\mathrm{a}}$, Daniel Grande ${ }^{\mathrm{b}}$ \\ ${ }^{a}$ Laboratoire Modélisation et Simulation Multi Echelle (MSME), UMR8208 CNRS, \\ Université Paris-Est, 5 boulevard Descartes, 77454 Marne la Vallée Cedex, France \\ ${ }^{\mathrm{b}}$ Institut de Chimie et des Matériaux Paris-Est (ICMPE), UMR 7182 CNRS, \\ Université Paris-Est, 2 rue Henri Dunant, 94320 THiais, France
}

\begin{abstract}
The paper presents a fully automated numerical tool for computing the intrinsic permeability of porous media from digital images which come from the modern imagery technique. The permeability is obtained by the homogenization process applied to a periodic rigid solid in which the fluid flow is described by the Stokes equations. The unit cell problem is solved by using the Fast Fourier Transform (FFT) algorithm, well adapted for the microstructures defined by voxels. Various 3-D examples are considered to show the capacity of the method. First, the case of flow through regular arrays of aligned cylinders or spheres are considered as benchmark problems. Next, the method is applied to some more complex and realistic porous solids obtained with assemblies of overlapping spherical pores having identical or different radii, regularly or randomly distributed within the unit cell. The use of FFT allows the resolution of high dimension problems and open various possibilities for computing the permeability of porous microstructures coming from x-ray microtomography.
\end{abstract}

Key words: Porous Media, Permeability, Homogenization, Fast Fourier Transform, Viscous Fluid.

Email addresses: ly@univ-mlv.fr (Hai-Bang Ly), vincent.monchiet@univ-paris-est.fr (Vincent Monchiet), grande@icmpe.cnrs.fr (Daniel Grande). 


\section{Introduction}

The determination of permeability of porous media in connection with microstructure morphology has been addressed with homogenization techniques based on asymptotic series expansion methods [1-3] or on energy principle and volume averaging $[4,5]$. Analytic methods have been first developed in the literature for solving the associated unit cell problem. For instance, some works use expansions along eigenfunctions [6-9], however such approaches are limited to some simple geometries corresponding to the flow through regular arrays of cylinders or spheres. The use of high-performance computers open number of possibilities for considering more realistic geometries, based on reconstructed porous cell or directly based on digital images obtained from X-ray microtomography. This also encourages the development of robust and efficient numerical tools for computing the effective permeability. For instance, standard numerical methods based on Finite Elements (FEM) or Finite Volumes have been often considered for computing the permeability of porous media [10-18]. The computation of permeability by means of the Boundary Element Method (BEM) has has been also proposed [22] or using the Lattice Boltzmann method [19-21]. When the microstructure is defined by voxels, the FEM can be used by considering a regular mesh with cubic elements. However the use of FFT type algorithms is more adapted for reducing the memory saving. Indeed, the method do not need the storage of stiffness or interaction matrices but only the storage of the nodal variables and the Fourier coefficients of the Green tensors. Moreover, the case of incompressible constituents (the fluid) does not requires specific treatments while, the FEM uses more sophisticated interpolation (for instance the "MINI" elements [23-25]) which generally increases the size of the system. In this paper, we apply the method based on Fast Fourier Transform for computing the permeability of complex 3-D microstructures defined by digital images [26]. Various problems and porous structures with increasing complexity are considered. First, the case of a flow around a cylinder and a sphere is considered as benchmark problems for which comparison with FEM or existing literature data are provided. Then, we construct more realistic porous media defined as assemblies of overlapping spherical voids to evaluate the capacity of this method.

\section{The FFT method in short}

We consider a unit cell containing an interconnected rigid solid $\Omega_{s}$ and an interconnected porous open set $\Omega_{f}$ saturated by a fluid. The flow is described by the Stokes equations and is generated by an applied pressure gradient denoted $\underline{G}$. The unit cell problem reads: 


$$
\begin{array}{rr}
\mu \Delta \underline{v}-\nabla p-\underline{G}=0 & \in \Omega_{f} \\
\operatorname{div} \underline{v}=0 & \in \Omega_{f} \\
\underline{v}=0 & \in S
\end{array}
$$

with the periodicity conditions for the velocity and the pressure. Let us denote by $\boldsymbol{\sigma}$, the stress defined by:

$$
\boldsymbol{\sigma}=\mu\left(\nabla \underline{v}+\nabla^{T} \underline{v}\right)-p \boldsymbol{I}
$$

where $\mathbf{I}$ is the two order identity tensor.

The cornerstone of the FFT numerical method is the reformulation of the system of partial differential equation in an integral equation which uses the Green operator and the convolution product. Such formulation of the problem is possible for the Stokes problem only by making a continuation by continuity of the local fields within the porous medium. Particularly, we consider a null velocity field within the solid phase. Alternatively, a null velocity field can be also recovered by taking the Stokes equation within $\Omega_{s}$ and a dynamic viscosity $\mu_{s}$ that is large enough behind that of the fluid. This viscosity can be interpreted as a penalty coefficient in order to recover the condition $\underline{v}=0$ within $\Omega_{s}$.

By doing so, the Stokes problem can be recast in an integral form [26]:

$$
\widehat{\sigma}=\widehat{\Omega} \cdot \underline{\hat{f}}-\widehat{\Delta}^{0}: \widehat{d}(\sigma)
$$

where the notation with the "hat" indicates that we use the Fourier transform of the corresponding quantity. In $(3), \widehat{\boldsymbol{d}}(\boldsymbol{\sigma})$ represents the Fourier transform of the strain rate tensor computed with the stress $\boldsymbol{\sigma}$. The strain rate being defined by:

$$
\boldsymbol{d}(\boldsymbol{\sigma})=A(x) *[\boldsymbol{\sigma}-p \mathbf{I}]
$$

with :

$$
A(x)=\frac{1}{2 \mu} \mathrm{I}_{\mathrm{f}}(x)+\frac{1}{2 \mu_{s}} \mathrm{I}_{\mathrm{s}}(x)
$$

where $\mathrm{I}_{\mathrm{f}}(x)$ and $\mathrm{I}_{\mathrm{S}}(x)$ are the characteristic functions of the fluid and the solid phase. $\mathrm{I}_{\mathrm{f}}(x)$ is equal to 1 if the point $\underline{x}$ belongs to the pore space and is equal to 1 otherwise while $\mathrm{I}_{\mathrm{s}}(x)=1-\mathrm{I}_{\mathrm{f}}(x)$.

In equation (3), $\widehat{\boldsymbol{\Delta}}^{0}$ is the complementary Green operator associated with the reference medium of dynamic viscosity $\mu_{0}$. Both $\widehat{\boldsymbol{\Delta}}^{0}$ and $\widehat{\boldsymbol{\Omega}}$ are explicitly known in the Fourier space. The Green tensor $\widehat{\boldsymbol{\Delta}}^{0}$ is defined by: 


$$
\widehat{\boldsymbol{\Delta}}^{0}=2 \mu_{0}\left[\boldsymbol{k}^{\perp} \otimes \boldsymbol{k}^{\perp}+\boldsymbol{k}^{\perp} \underline{\bar{\otimes}} \boldsymbol{k}^{\perp}\right]
$$

for $n \neq 0$ and $\widehat{\boldsymbol{\Delta}}_{n}^{0}=0$ for $n=0$ and where $\boldsymbol{k}$ and $\boldsymbol{k}^{\perp}$ are given by:

$$
\boldsymbol{k}=\frac{1}{|\underline{\xi}|^{2}} \underline{\xi} \otimes \underline{\xi}, \quad \boldsymbol{k}^{\perp}=\mathbf{I}-\boldsymbol{k}
$$

The components of the third order tensor $\widehat{\Omega}$ are:

$$
\widehat{\Omega}_{i j k}(\boldsymbol{\xi})=\frac{i}{|\xi|}\left[2 \bar{\xi}_{i} \bar{\xi}_{j} \bar{\xi}_{k}-\delta_{i j} \bar{\xi}_{k}-\delta_{i k} \bar{\xi}_{j}-\delta_{j k} \bar{\xi}_{i}\right], \overline{\boldsymbol{\xi}}=\boldsymbol{\xi} /|\xi|
$$

In equation (3), $\underline{f}$ is given by:

$$
\underline{f}= \begin{cases}\underline{G} & \text { in } \Omega_{f} \\ -\frac{c_{f}}{c_{s}} \underline{G} & \text { in } \Omega_{s}\end{cases}
$$

where $c_{f}$ and $c_{s}$ denote the volume fraction of the fluid and the solid phase respectively.

$\underline{f}$ can be interpreted as a source term in the Stokes equation which is equal to the the applied pressure gradient in the fluid phase. A constant value for $f$ is considered in the solid phase and can be interpreted as the drag force due to the fluid flow around the solid $\Omega_{s}$. This drag force is computed in order to obtain the equilibrium of the force applied to the unit cell (see [26] for more details).

The solution of the integral equation (3) is solved by means of the following iterative process:

$$
\widehat{\boldsymbol{\sigma}}^{i+1}=\widehat{\boldsymbol{\sigma}}^{i}-\widehat{\boldsymbol{\Delta}}^{0}: \widehat{\boldsymbol{d}}\left(\boldsymbol{\sigma}^{i}\right)
$$

which can be interpreted as a fixed point iterative method associated with the linear system (3). The iterative scheme (10) is initialized with :

$$
\widehat{\boldsymbol{\sigma}}^{i=1}=\widehat{\Omega} \cdot \underline{\hat{f}}
$$

Once the convergence is achieved, one can compute the velocity field from the strain rate tensor $\widehat{\boldsymbol{d}}$ :

$$
\underline{\widehat{v}}=\widehat{\boldsymbol{d}}: \widehat{\Omega}
$$


for all $\underline{\xi} \neq 0$. The velocity field is defined by its Fourier coefficients for all values of $\xi$ except for $\xi=0$. It means that the velocity field is defined up to an added constant that represents its mean value over the volume of the cell. This constant is identified by considering the condition $\mathbf{v}=0$ in the solid phase. Let us denote by $\underline{v}^{*}$ the velocity field computed from equation (12) for any $\underline{\xi} \neq 0$ and $\underline{\widehat{v}}^{*}=0$ for $\underline{\xi}=0$. This velocity field has a null volume average over $\Omega$ and it is constant within $\Omega_{s}$. The total velocity field $\underline{v}$ is then the sum of $\underline{v}^{*}$ and a constant velocity $\underline{V}$ which represents the average of $\underline{v}$ over the volume $\Omega$ :

$$
\underline{\widehat{v}}=\underline{\widehat{V}}+\underline{\widehat{v}}^{*}
$$

Since $\underline{\hat{v}}$ is null within $\Omega_{s}$, the macroscopic velocity field is given by $\underline{V}=-\underline{v}^{*}$ in $\Omega_{s}$. From a practical point of view, the condition $\underline{v}(x)=0$ is not rigorously verified everywhere in $\Omega_{s}$. The macroscopic velocity is then computed by considering one point taken within $\Omega_{s}$ or by taking the average of $\underline{v}^{*}$ over the volume $\Omega_{s}$. When one point in $\Omega_{s}$ is considered, this point must not be taken so closed to the surface of the solid. Indeed, at the vicinity of the surface, the occurrence of the Gibbs phenomenon, that is inherent with the use of Fourier series, could affect the accuracy of the solution.

For the implementation of the iterative scheme we put $1 / \mu_{s}=0$. Obviously a very high value is considered for the penalty coefficient, however the numerical application shows that with the value $1 / \mu_{s}=0$ a good rate of convergence is observed. Moreover, we choose $\mu_{0}=2 \mu$ that is optimal for the convergence of the algorithm.

\section{Discretization of the problem}

Following the approach developed for composite materials $[27,35]$, the problem is discretized along a regular grid consisting of $N \times N \times N$ voxels. The nodal variables are the components of the stress that depends on 6 independent components. The constituents (the solid and the fluid) distribution within the unit cell is accounted in the iterative process by the characteristic function $I_{f}$ which appears in relation (4). At each node of the regular grid, the value 0 or 1 is assigned depending on either this point is in the solid or it is in the fluid phase. After discretization, the convolution product between $I_{f}$ and the stress is made by means of the FFT algorithm. In equation (10), all the components of the Green tensor are represented by their Fourier coefficients in the real space in terms of the discrete wave vectors $\underline{\xi}_{n}$ given by

$$
\underline{\xi}_{n}=2 \pi n \underline{\zeta}, \quad n=-N . . N-1, \quad \zeta_{i}=\frac{1}{h_{i}}
$$


and $h_{1}, h_{2}, h_{3}$ are the dimensions of the cell along each directions of the cartesian frame. Obviously, the problem is discretized along each space direction, this would involve the use of two indices $n_{1}$ and $n_{2}$ for 2-D problems and three for 3-D ones. However, only one indice $n$ is used for simplicity. The product between the components of the strain rate tensor and those of the Green operator just consists in the Hadamard product of tables of dimension $N \times N \times N$. The discretized algorithm is:

1: $\quad$ compute $I_{f n}=I_{f}\left(\underline{x}_{n}\right)$

2: $\quad$ compute $\boldsymbol{\Omega}_{n}=\boldsymbol{\Omega}\left(\underline{\xi}_{n}\right)$ following Eq. (8)

3 : $\quad$ compute $\widehat{\boldsymbol{\Delta}}_{n}^{0}=\widehat{\boldsymbol{\Delta}}^{0}\left(\underline{\xi}_{n}\right)$ following Eq. (6)

4: compute $\underline{f}_{n}=-\frac{1}{c_{s}} \underline{G} I_{f n}$

$5: \quad \underline{f}_{n}=\mathrm{FFT}\left(\underline{f}_{n}\right)$

$6: \quad$ initialize $\widehat{\boldsymbol{\sigma}}_{n}^{1}=\widehat{\boldsymbol{\Omega}}_{n} \cdot \widehat{\widehat{f}}_{n}$

7: $\quad$ while test $<\epsilon d o$

$8: \quad \boldsymbol{\sigma}_{n}^{i}=\mathrm{FFT}^{-1}\left(\widehat{\boldsymbol{\sigma}}_{n}^{i}\right)$

$9: \quad p_{n}^{i}=\operatorname{tr}\left(\boldsymbol{\sigma}_{n}^{i}\right) / 3$

$10: \quad \boldsymbol{d}_{n}=\frac{1}{\mu} I_{f n}\left(\boldsymbol{\sigma}_{n}^{i}-p_{n}^{i} \boldsymbol{I}\right)$

$11: \quad \widehat{\boldsymbol{d}}_{n}^{i}=\operatorname{FFT}\left(\boldsymbol{d}_{n}^{i}\right)$

$12: \underline{\underline{v}}_{n}^{i}=\widehat{\boldsymbol{d}}_{n}^{i}: \widehat{\Omega}_{n}$

$13: \quad$ test $=\sqrt{\sum_{n}\left\|\underline{\widehat{v}}_{n}^{i}-\underline{\widehat{v}}_{n}^{i-1}\right\|^{2}}$

$14: \quad \widehat{\boldsymbol{\sigma}}_{n}^{i+1}=\widehat{\boldsymbol{\sigma}}_{n}^{i}-\widehat{\boldsymbol{\Delta}}_{n}^{0}: \widehat{\boldsymbol{d}}_{n}$

15: end while

For simplicity, we have used the following notations for any variable $F(\underline{x})$ and its Fourier transform $\widehat{F}(\underline{\xi}): F_{n}=F\left(\underline{x}_{n}\right)$ and $\widehat{F}_{n}=\widehat{F}\left(\underline{\xi}_{n}\right)$.

At step 7, we use a convergence test based on the norm of the difference between the local velocity computed at the iteration $i$ and $i-1$ and we choose $\epsilon=10^{-4}$. It is noteworthy that another test has been considered in previous works to check the convergence of the FFT algorithms. For instance, a convergence test based on the compatibility for the strain has been used [36]. However, since comparisons are provided with the solution obtained with the FEM, we have chosen a convergence test applicable to both methods.

From a practical point of view, the permeability is computed by taking $\mu=1$ and we apply the pressure gradient $\underline{G}=-\underline{e}_{i}$ for $i=1,2,3$ where $\left(\underline{e}_{1}, \underline{e}_{2}, \underline{e}_{3}\right)$ 
is the unit vector of the cartesian basis. With this choice, the average of the velocity field, $V_{i}=<v_{i}>_{\Omega}$ is exactly $V_{i}=K_{i j}$ when $\underline{G}=-\underline{e}_{j}$ is prescribed to the system.

In any case considered in the next of the paper, the total number of nodes is increased and the permeability is computed for different values of $N$ in order to evaluate the accuracy of the solution. Considering the maximum possible memory occupancy of our computer cluster, the resolution cannot exceed $1024 \times 1024 \times 1024$ voxels when using the FFT method.

Calculations were performed using MATLAB 2011 on an AMD Opteron processor, $2.5 \mathrm{GHz}, 1024 \mathrm{~GB}$ RAM and 64 cores.

\section{Benchmark problems}

Stokes flow through arrays of cylinders and spheres has been often considered in many studies in the literature. For instance, the permeability of porous media with cylinders has been investigated by Sparrow and Loeffler [37], Banerjee and Hadaller [38], Sangani and Acrivos [6], Drummond and Tahir [39], Larson and Higdon [40,41], Wang [9] and Idris [42]. The numerical implementation of the Stokes problem through an array of rigid spheres has been provided by Sangani and Acrivos [7], Barrere et al. [10], Chapman and Higdon [43], Kadaksham et al. [44]. Note that some works deal with the extension of the self-consistent in the field of periodic homogenization method to derive analytic expressions for the permeability $[45,46]$ but the formula are restricted to some particular microstructures corresponding to porous media with rigid cylinders or spheres. Obviously, a unit cell containing a rigid cylinder or sphere cannot mimic realistically a porous solid and these two examples are only considered here as benchmark problems. The solutions obtained with the FFT method are compared with the results obtained by Sparrow and Loeffler [37], Wang [9] for the cylinder and those obtained by Sangani and Acrivos [7] for the sphere. The computations are performed on a dimensionless unit cell (having the dimension 1 along each direction), leading then to the computation of a dimensionless permeability. For the FEM, we use cubic elements and the interpolation is made with the MINI elements following a method previously proposed [25]. In the cubic elements, the solid or the fluid phase is assigned. All the solid voxels are eliminated in the resulting linear system which advantageously reduce the size of the problem. The adherence condition on the surface of the solid is then accounted with penalty coefficients. The total number of voxels considered with the FEM is $192 \times 192 \times 192$ voxels, which corresponds to the maximum possible memory occupancy. A comparison with available data is provided in the case of the flow through an array of rigid cylinders or spheres, the corresponding unit is represented in Figure 1. The transversal and longitudinal components of the permeability tensor are successively com- 
puted by applying a pressure gradient parallel or orthogonal to the cylinder axis. The results for the transversal and longitudinal permeability are shown in figures 2 and 3, respectively. The FFT solutions are compared with those of Wang [9], Sparrow and Loeffler [37]. A good agreement is observed between our numerical values and those reported in the literature.
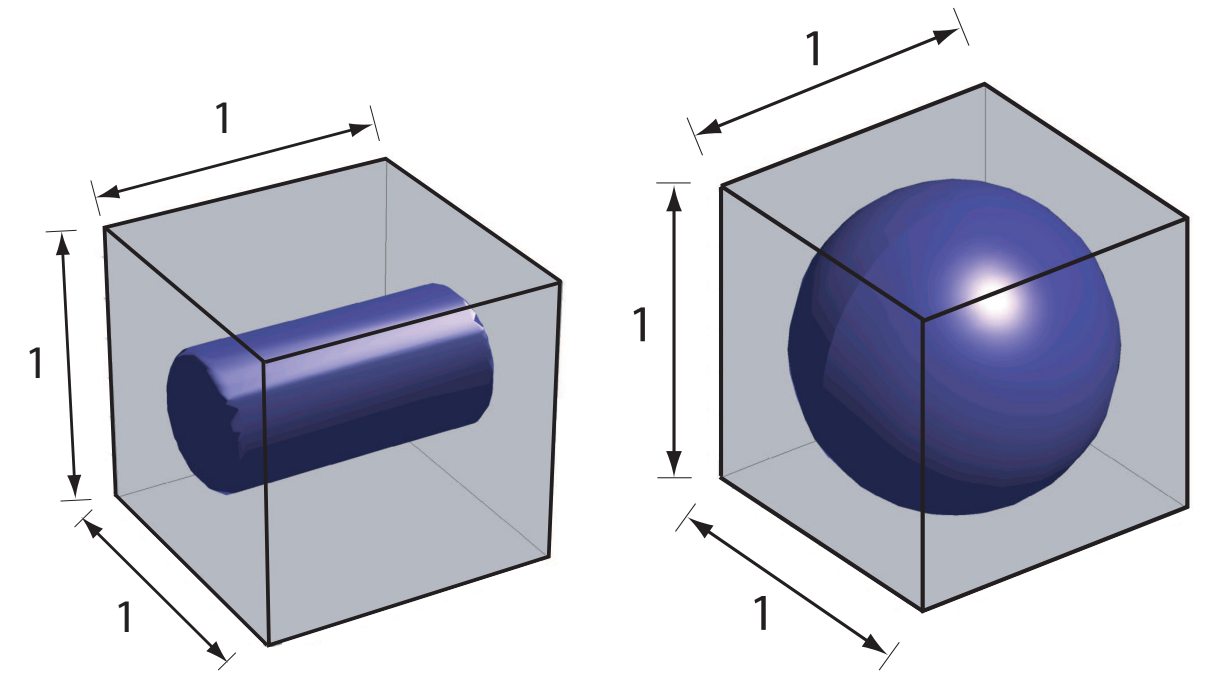

Fig. 1. Unit cell containing a rigid cylinder or sphere

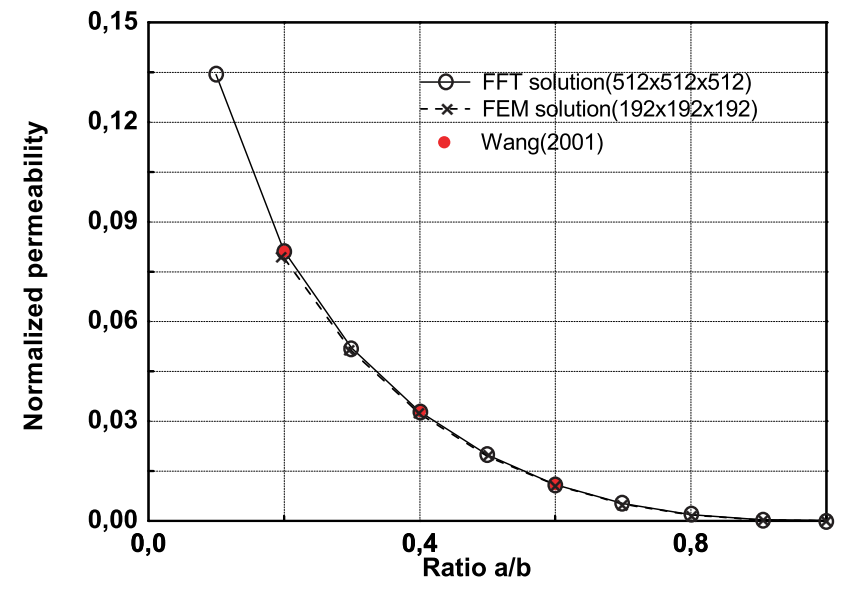

Fig. 2. Dimensionless transversal permeability for the flow through a regular array of cylinders. Comparison of the FFT solution, the FEM solution and with the results obtained by Wang [9]. $R$ is the radius of the cylinder or the sphere, and $L$ is the half-length of the unit cell. 
Table 1

\begin{tabular}{|l|l|l|l|}
\hline Radius & FFT $\left(512^{3}\right)$ & FFT $\left(128^{3}\right)$ & FEM $\left(128^{3}\right)$ \\
\hline 0.1 & 0.134310 & 0.133383 & 0.131609 \\
0.2 & 0.081148 & 0.080984 & 0.079120 \\
0.3 & 0.051926 & 0.051386 & 0.051078 \\
0.4 & 0.032864 & 0.032476 & 0.032255 \\
0.5 & 0.019855 & 0.019600 & 0.019366 \\
0.6 & 0.010921 & 0.010692 & 0.010769 \\
0.7 & 0.005143 & 0.005049 & 0.004966 \\
0.8 & 0.001812 & 0.001699 & 0.001782 \\
0.9 & 0.000308 & 0.000279 & 0.000302 \\
\hline
\end{tabular}

Comparison of the intrinsic permeability of transverse flow through array of cylinders computed with the FFT method and the FEM.

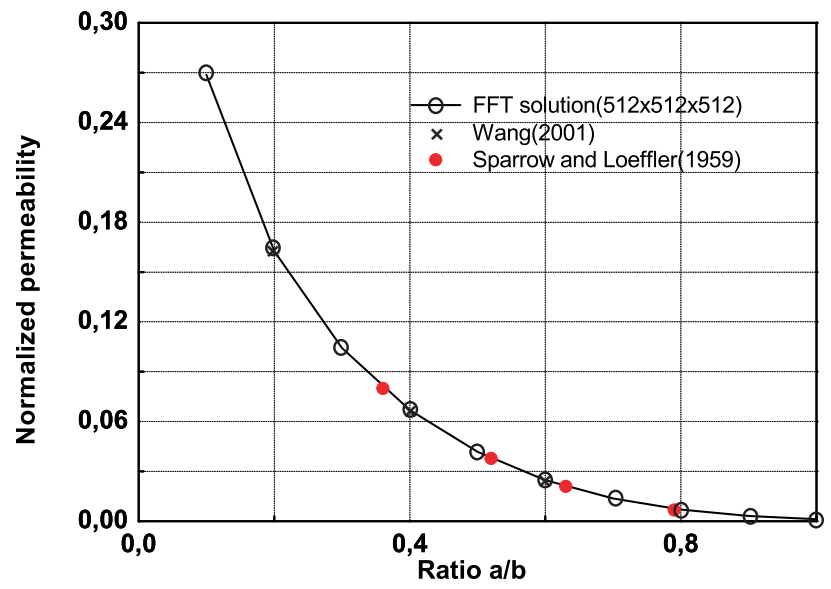

Fig. 3. Dimensionless longitudinal permeability for the flow through a regular array of cylinders. Comparison of the FFT solution with the results obtained by Sparrow and Loeffler [37], and Wang [9].

The results obtained with the FFT method and the FEM are now qualitatively compared on figures 1 for the transverse flow through the array of cylinders and for various values of the Radius. The FFT-based data are provided for the resolution $128 \times 128 \times 128$ and for $512 \times 512 \times 512$ while, the results obtained with FEM are given for the resolution $128 \times 128 \times 128$.

We now consider numerical applications in the case of a regular array of rigid spheres. The convergence of the FFT solution with the grid refinement is provided in figure 4 for the radius $R=0.15, R=0.25$ and $R=0.35$, and it is also compared with FEM solutions. A good agreement between the FFT and the FEM methods is observed for the largest value of the radius $(R=0.35)$. 
There is a slight difference for the radius $R=0.25$, that is more significant for $R=0.15$. These differences are essentially attributed to the discretization error. Indeed, the sphere being approximated by means of cubic elements, the error is higher for the smaller radius. This is a well known limitation of the methods which use a regular grid.

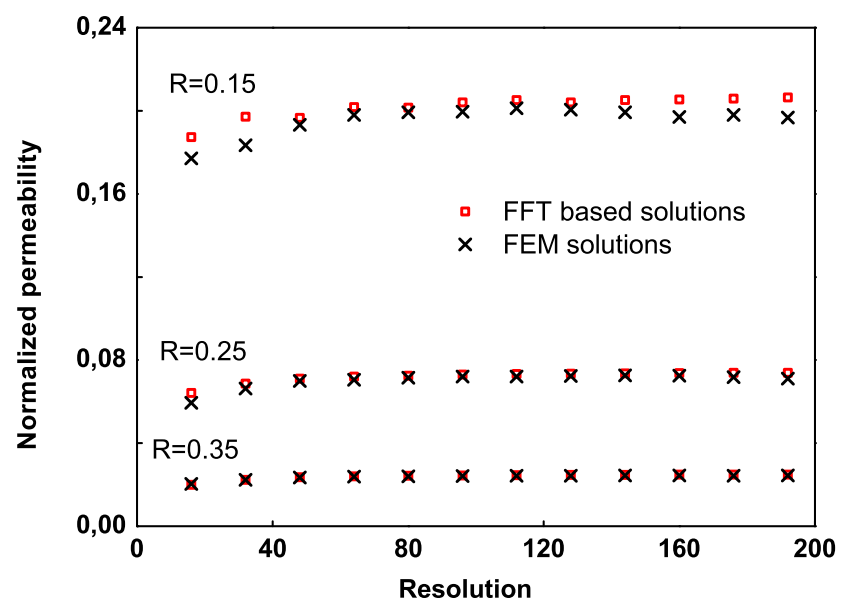

Fig. 4. Dimensionless permeability for the flow through a regular array of sphere of radius $R=0.15, R=0.25$ and $R=0.35$. Comparison between the FFT and the FEM solution.

The FFT solution (with $512 \times 512 \times 512$ voxels) and the FEM one (with $192 \times 192 \times 192$ voxels) are compared with the data provided by Sangani and Acrivos [7] in Table 1. Various values of the radius of the sphere have been considered in the range $[0.1,1]$. It is observed that the FFT solutions are in a better agreement with the data of Sangani and Acrivos [7] than the FEM solution. The error between the FFT method and the results of Sangani and Acrivos [7] vary from $0.6 \%$ (in the case $R=0.1$ ) to $0.13 \%$ (in the case $R=1$ ). This was expected, since the FFT solution was computed with a more refined grid than the FEM solution. Obviously, the results would be compared with the same discretization, however here we compare the solutions obtained by these two methods and the maximum possible memory occupancy of the computer. This clearly shows that the FFT is computationally more attractive for reducing the memory and hence its application to the problems of high dimensions. Such comparison has been already provided in the literature but in the context of composite materials [28]. The calculation times needed for solving the FFT method are provided on table 3 . The calculation time depends on the number of iterations needed to obtain the convergence. Particularly, it must be observed that for the flow around the rigid sphere, the number of iteration increases with the radius of the sphere. 
Table 2

\begin{tabular}{|c|c|c|c|}
\hline R & FFT & FEM & Sangani and Acrivos [7] \\
\hline 0.1 & 0.905109 & - & 0.911070 \\
0.2 & 0.380631 & - & 0.382190 \\
0.3 & 0.207387 & 0.196734 & 0.208050 \\
0.4 & 0.122899 & - & 0.123270 \\
0.5 & 0.074448 & 0.070996 & 0.074668 \\
0.6 & 0.044340 & - & 0.044501 \\
0.7 & 0.025136 & 0.024461 & 0.025246 \\
0.8 & 0.013121 & - & 0.013197 \\
0.85 & 0.009098 & - & 0.009151 \\
0.9 & 0.006140 & - & 0.006153 \\
0.95 & 0.003980 & - & 0.004003 \\
1 & 0.002487 & - & 0.002520 \\
\hline
\end{tabular}

Dimensionless permeability for the flow through a regular array of sphere with radius lying in the range $[0.1,1]$. Comparison between the FFT solution, the FEM solution and the results of Sangani and Acrivos [7].

Table 3

\begin{tabular}{|l|l|}
\hline Radius & Time $(\mathrm{s})$ \\
\hline 0.1 & $869(\sim 14 \mathrm{~min})$ \\
0.2 & $1001(\sim 17 \mathrm{~min})$ \\
0.3 & $1126(\sim 19 \mathrm{~min})$ \\
0.4 & $1139(\sim 19 \mathrm{~min})$ \\
0.5 & $1190(\sim 20 \mathrm{~min})$ \\
0.6 & $1404(\sim 23 \mathrm{~min})$ \\
0.7 & $1691(\sim 28 \mathrm{~min})$ \\
0.8 & $2002(\sim 33 \mathrm{~min})$ \\
0.9 & $2920(\sim 49 \mathrm{~min})$ \\
\hline
\end{tabular}

Computation time (in seconds) with the FFT-based algorithm in the case of the flow around the rigid sphere of $R$.

\section{Advanced numerical results}

In this section, we consider more realistic porous cells which can mimic for instance metal foams or porous polymer solids [29]. Various configurations are obtained by an assembly of overlapping spherical pores. For most studies on composite materials, the hypothesis of non-overlapping inclusions is consid- 
ered, while in the present case, it is necessary to consider overlapping spheres in order to obtained a network of interconnected pores. In this section we are only interested by the evaluation of the capacity of the FFT method to handle the Stokes flow problem through complex porous microstructures and to provide some comparisons with the FEM method. The next step will concern the application to idealized reconstructed unit cells or to digital images obtained from microtomography. As for example, polymeric PHEMA-based porous materials are elaborated with spherical particulates and the porosity is generated with porogenic solvents (see for instance Le Droumaguet et al. 2014). These porous materials have demonstrated some potential or promising results in the development of devices for drug delivery or scaffolds for tissue engineering applications (see for example Liu et al. 2004, Lee et al. 2007). The use of idealized tridimensional porous cell made up of overlapping spherical pores is adapted for this kind of materials and the mass transfer properties must be studied on reconstructed porous cell knowing some the characteristic size of the particulates and the porosity. As another example, the acoustic properties of metal foams are studied can be studied by performing calculations on idealized unit cells (see for instance Hoang and Perrot [32,33]). Metal foams are often used to increase sound absorption in aeronautics and building construction. The determination of the dynamic permeability is the cornerstone of the acoustic approaches of porous media and can be determined using the FFT approach developed in Nguyen et al. [34].

As a first example, we represent, in figures 5 and 6 , a regular array of spherical overlapping voids arranged along a cubic lattice. Figure 5 represents, at the left, a unit cell having the dimension 2 and containing a body-centered cubic (BCC) array of spherical cavities with the radius $R=0.5$. Each spheres intersect another one along the diagonals of the cube. The resulting irreducible unit cell of the porous medium is represented in figure 5, at the right. The porosity for the BCC array is $\phi=0.939$.

Another example is given in figure 6 . At the left, we represent a face-centered cubic (FCC) structure of spherical voids. At the right, the irreducible unit cell of the porous medium is represented for the FCC structure. The radius of the spherical voids is $R=0.375$. The porosity for the FCC array is $\phi=0.858$. The results for the permeability are shown in figure 7 and 8 . The permeability, computed with the FFT method and the FEM, is plotted as function of the grid refinement. 

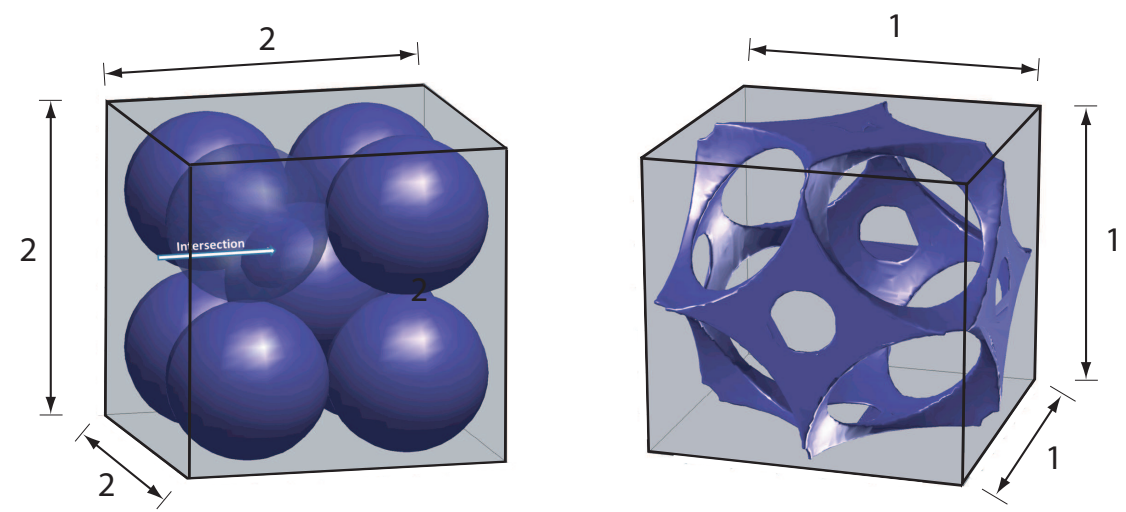

Fig. 5. Body-centered array of spherical voids. At the left: the voids distribution, at the right: the rigid porous solid.
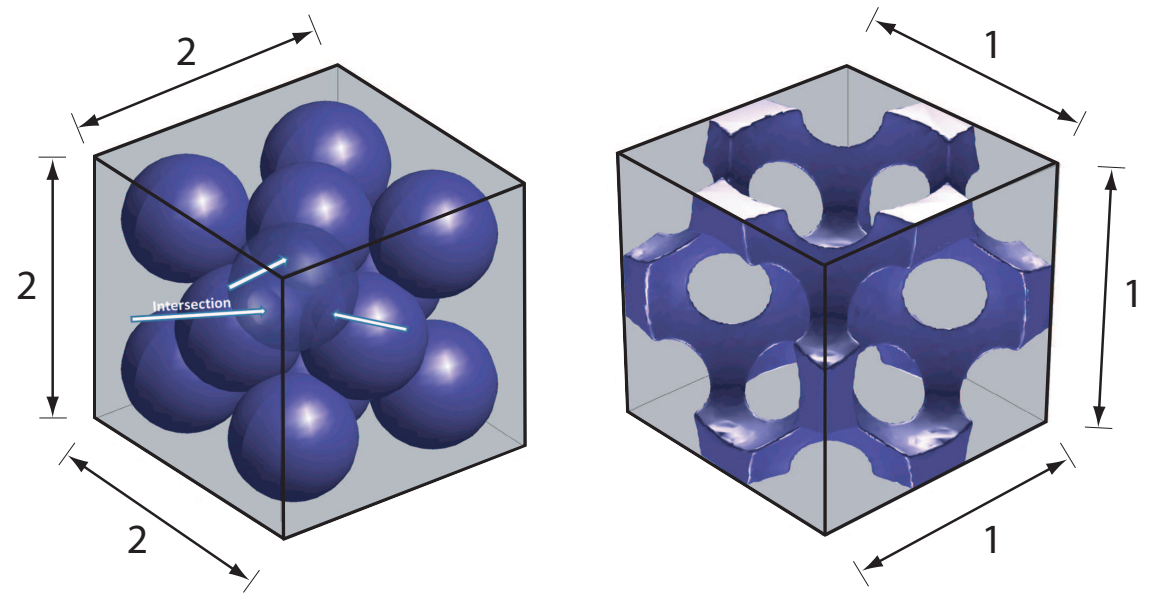

Fig. 6. Face-centered array of spherical voids. At the left: the voids distribution, at the right: the rigid porous solid. 


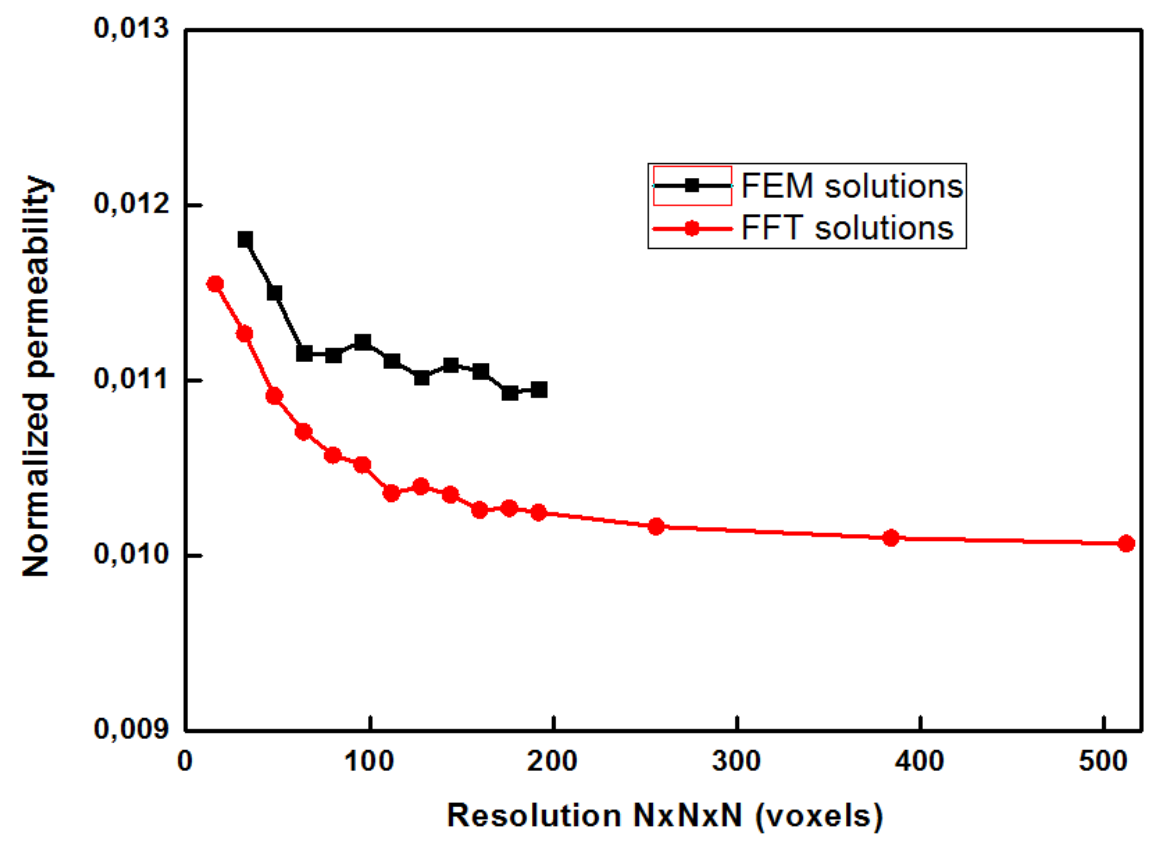

Fig. 7. Dimensionless permeability for the flow through a body-centered array of spherical voids.

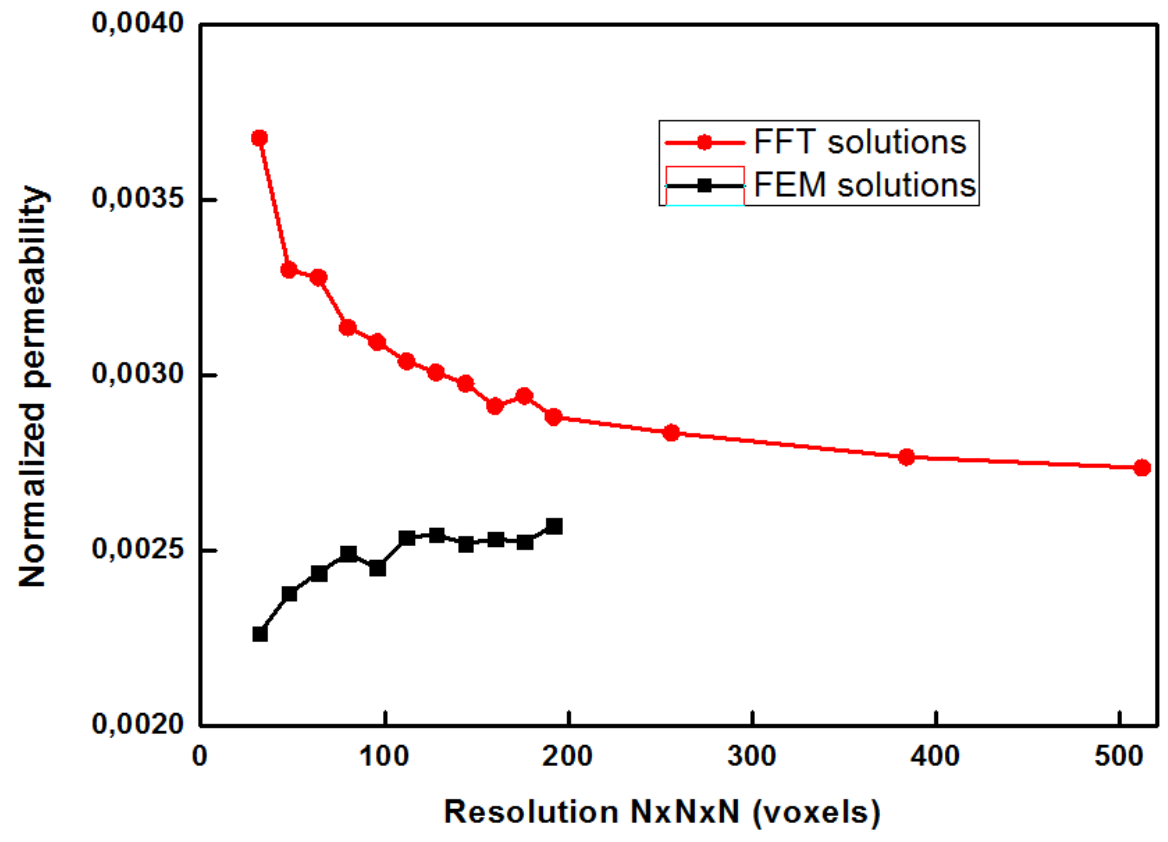

Fig. 8. Dimensionless permeability for the flow through a face-centered array of spherical voids.

We now consider a more complex porous medium (Fig. 9) defined by spherical voids with different sizes : a larger void located at the center of the cubic cell having the radius $R_{1}=0.52$ and 376 smaller voids having the radius $R_{2}=0.06$. 
Each smaller void overlaps with its neighboring void and some small voids also overlaps the larger one. By doing so, the fluid domain is then ensured to be interconnected. The porosity for this microstructure is $\phi=0.915$. In figure 10, we consider a second example corresponding to a random distribution of the smaller voids. The unit cell contains 536 voids with a radius comprised between 0.06 and 0.08 randomly distributed, and a large void still located at the center of the cell and having the radius $R=0.52$. The porosity for this last example is $\phi=0.871$.

The process used to construct the unit cell is the following: We build one eighth of the unit cell that we duplicate by symmetry with respect to three orthogonal planes (Ox1x2, Ox1x3 and Ox2x3 in the cartesian frame). By doing so, the resulting microstructure is then periodic. The construction of the eighth of the unit cell is made in two steps. In the first one, we put the larger sphere of radius $R=0.52$ at the center of the unit cell. In the second step we put all the smaller voids and we use numerical tests. The radius of each small void is randomly considered in the range $[0.06,0.08]$ and its position is randomly chosen in the range $[0,0.5] \times[0,0.5] \times[0,0.5]$. Numerical tests are used to ensure that for each occurrence, the smaller void cross at least one other smaller void or the larger void. This verification is needed in order to obtain an interconnected microstructure.

The dimensionless permeability for these two microstructures is represented in figures 11 and 12. In the case of the regular arrangement of smaller voids (and also for the BCC and the FCC arrays), the permeability is isotropic and is defined by only one coefficient. However, in the particular case corresponding to random distribution of smaller voids, the permeability tensor is anisotropic. In fact, in figure 12, we only represent the component $K_{11}$ by applying a pressure gradient along the direction $O x_{1}$. The microstructure is however quasi-isotropic, the off-diagonal coefficients of the permeability tensor are close to 0 and the terms on the diagonal take very close values. The lower convergence observed on figure 12 will probably be attributed to the tortuosity of the porous medium with random pores compared to the case of regularly aligned pores. This tortuosity would introduce some complexity in the local field that an accurate description would require higher order Fourier series expansions (that is obtained by increasing of the resolution of the image). The application of the FEM to the case of random distributions of overlapping sphere fails due to the voxelization of the image. Indeed, for such microstructure the smaller voids must contain a sufficient number of voxels such that the problem would be numerically solved. If a least the number of vowels is not sufficient, the fluid flow circulation is blocked in the smaller voids. When the FFT method is used, the results are only provided for a resolution equal or higher than $128^{3}$. When the FEM is used the resolution of the linear system is made with an iterative method whose convergence greatly depends on the geometry of the porous medium. Particularly, for the porous cell with a random distribution of spherical voids, the time computation has found to be prohibitive. 

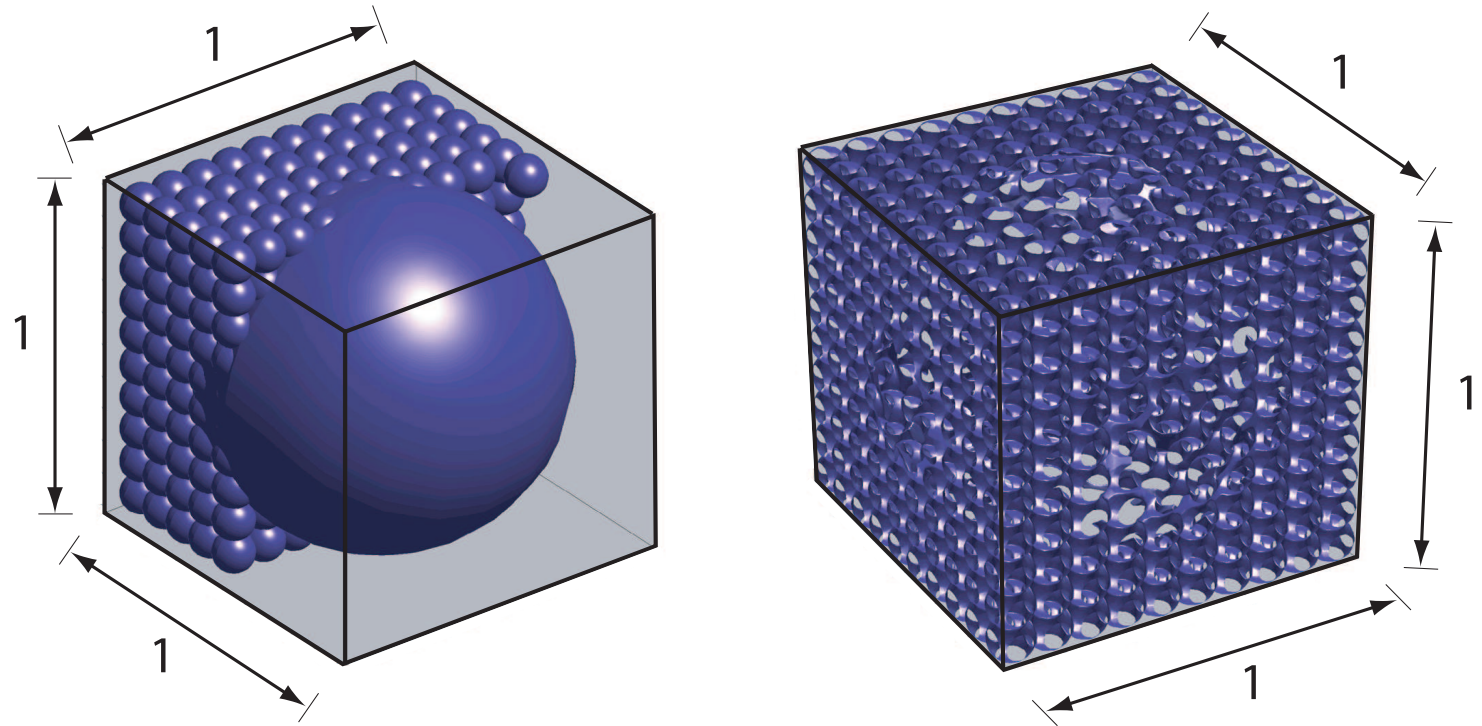

Fig. 9. Porous material with 376 regularly aligned small voids and one larger void located at the center of the unit cell. At the left: the pore positions, at the right: the rigid porous solid.
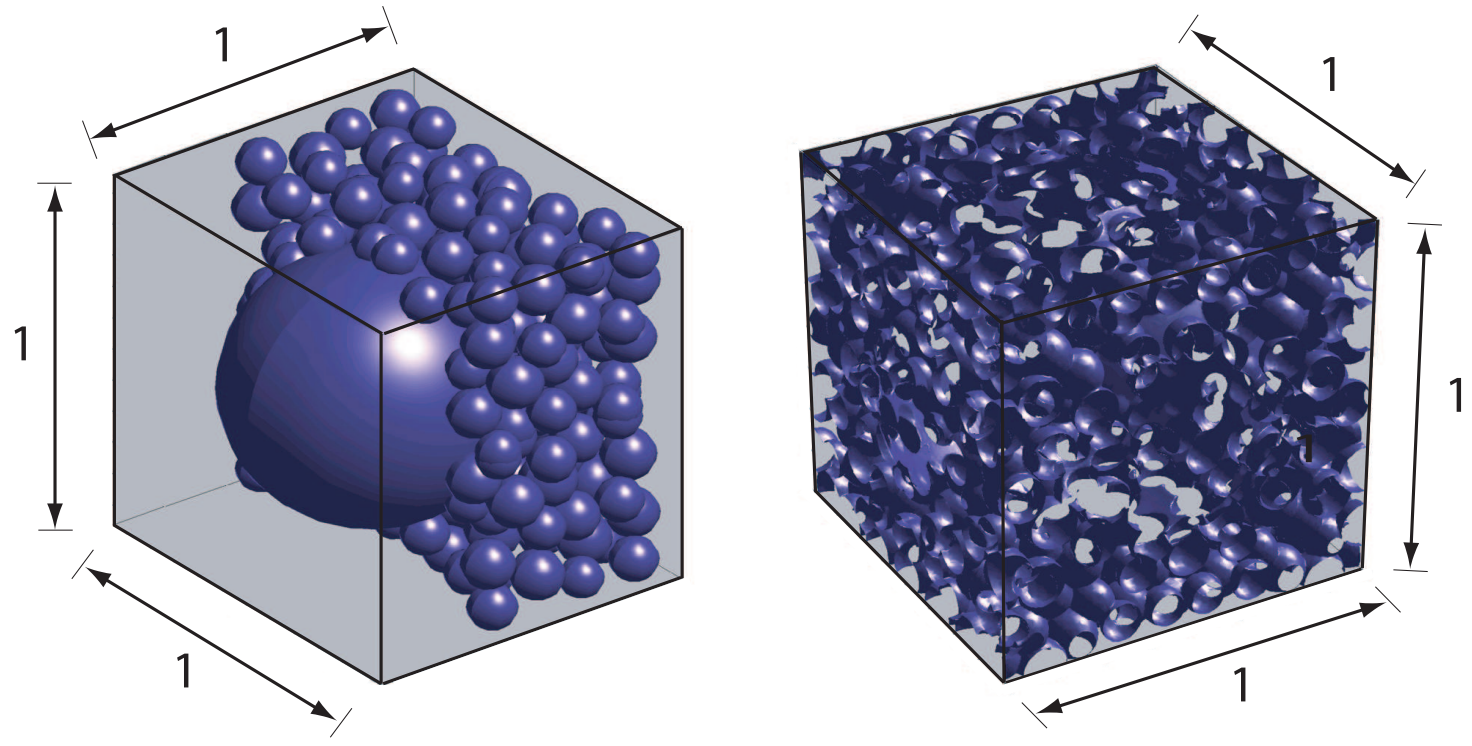

Fig. 10. Porous material with 536 randomly distributed small voids and one larger void located at the center of the unit cell. At the left: the pore positions, at the right: the rigid porous solid. 


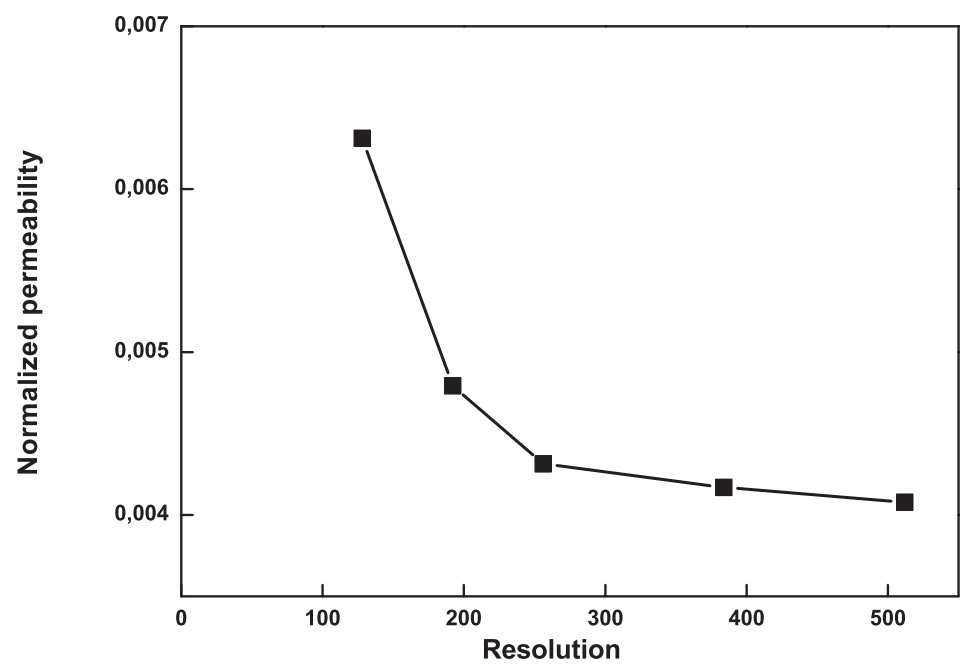

Fig. 11. Dimensionless permeability for the flow through the microstructure given in Fig. 9

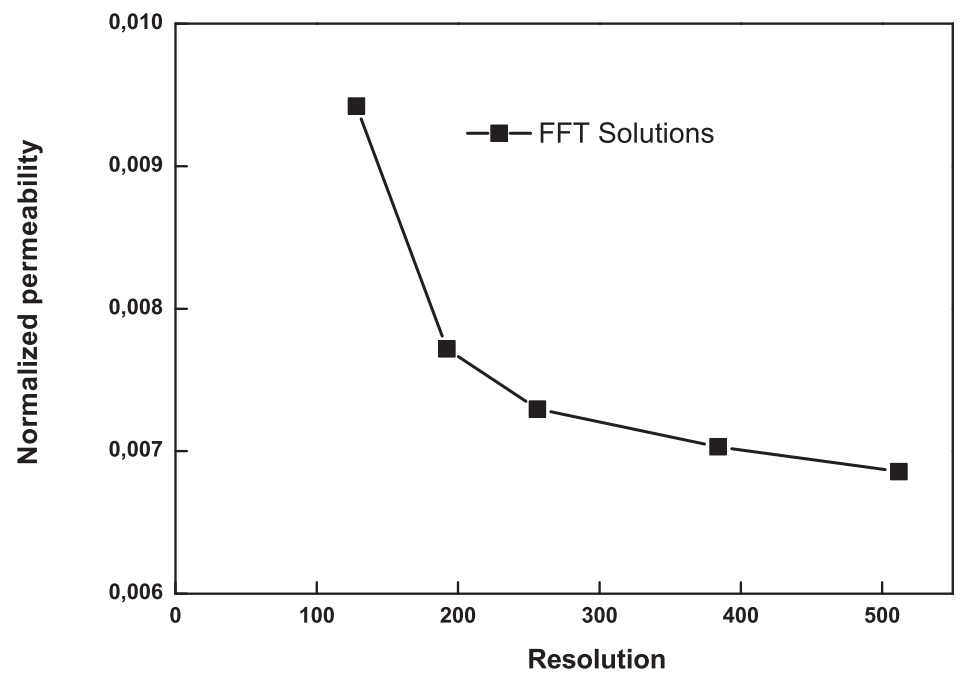

Fig. 12. Dimensionless permeability for the flow through the microstructure given in Fig. 10

For completeness, in Tables 2-5, we also provide the values of the permeability for the flow through different microstructures depicted in figures 5, 6, 9 and 10 and for the values of resolution $128^{3}, 256^{3}, 384^{3}$ and $512^{3}$. Just as indication, we also provide the relative error along formula given in Table 2, to evaluate the accuracy of the solution and the rate of convergence with respect to the resolution. It is observed that the relative error decreases when the resolution increases, that is expected. However, at a given spatial resolution, the error significantly differs from one microstructure to another. In particular, the the relative error is the lowest for the BCC array of pores (see Fig. 5) that is close to 0.1 percent for the spatial resolution of $512^{3}$. The relative error is the highest for the last example corresponding to the random distribution of small 
Table 4

\begin{tabular}{|c|c|c|}
\hline resolution & permeability & relative error \\
\hline $128^{3}$ & 0.010398 & - \\
$256^{3}$ & 0.010169 & $\frac{K_{64}-K_{128}}{K_{64}+K_{128}}=0.01113$ \\
$384^{3}$ & 0.010104 & $\frac{K_{128}-K_{192}}{K_{128}+K_{192}}=0.00321$ \\
$512^{3}$ & 0.010072 & $\frac{K_{192}-K_{256}}{K_{192}+K_{256}}=0.00159$ \\
\hline
\end{tabular}

Values of permeability and relative error as function of the resolution for the BCC array of spherical pores

Table 5

\begin{tabular}{|c|c|c|}
\hline Resolution & permeability & relative error \\
\hline $128^{3}$ & 0.003009 & - \\
$256^{3}$ & 0.002837 & 0.02942 \\
$384^{3}$ & 0.002768 & 0.01231 \\
$512^{3}$ & 0.002737 & 0.00563 \\
\hline
\end{tabular}

Values of permeability and relative error as function of the resolution for the FCC array of spherical pores

Table 6

\begin{tabular}{|c|c|c|}
\hline Resolution & permeability & relative error \\
\hline $128^{3}$ & 0.006313 & - \\
$256^{3}$ & 0.004314 & 0.18811 \\
$384^{3}$ & 0.004170 & 0.01697 \\
$512^{3}$ & 0.004078 & 0.01115 \\
\hline
\end{tabular}

Values of permeability and relative error as function of the resolution for the problem with a large pore and smaller aligned pores

voids depicted on Fig. 10 that is close to 1.2 percents for the spatial resolution of $512^{3}$. It is also interesting to note that that the relative error given on table 4 and 5 are quite similar, even though the results corresponds to a regular or a random distribution of spherical voids. It seems that the tortuosity, which is more important for the random distribution, do not really affect the rate of convergence with the spatial distribution. The calculation times needed are provided on table 8 for the resolution $512 \times 512 \times 512$.

\section{Conclusion}

In the present paper, we have employed a numerical FFT-based method for computing the permeability of periodic porous media from digital images which come from modern imagery devices. Different configurations have been 
Table 7

\begin{tabular}{|c|c|c|}
\hline Resolution & permeability & relative error \\
\hline $128^{3}$ & 0.009423 & - \\
$256^{3}$ & 0.007296 & 0.12722 \\
$384^{3}$ & 0.007032 & 0.01842 \\
$512^{3}$ & 0.006856 & 0.01267 \\
\hline
\end{tabular}

Values of permeability and relative error as function of the resolution for the problem with a large pore and smaller randomly distributed pores

Table 8

\begin{tabular}{|l|l|}
\hline Configurations & Time $(\mathrm{s})$ \\
\hline BCC & $144149(\sim 1.7$ day $)$ \\
FFC & $81317(\sim 0.94$ day $)$ \\
376 regularly aligned & $163826(\sim 1.9$ day $)$ \\
536 randomly distributed & $643283(\sim 7.44$ days $)$ \\
\hline
\end{tabular}

Computation time (in seconds) with the FFT-based algorithm and for the different problems and with the resolution $512 \times 512 \times 512$ voxels

tested to show the capacity of the method and the accuracy of its solution. First, benchmark problems, consisting of regular arrays of rigid cylinders or spheres have been considered. The results have been compared to those coming from the literature and show a good accuracy of the FFT-based solution. Advanced numerical results have been then considered. Some microstructures are constructed with an assemblies of overlapping spherical voids that can mimic more realistically the real microstructure of some human-made porous materials. First, porous media are obtained by considering regularly distributed spheres along a body-centered cubic (BCC) or face-centered cubic (FCC) structures. More complex microstructure are then studied by considering porous media having different pore size which are regularly or randomly distributed. In each case, the results show a good convergence with the grid refinement used by the FFT algorithm.

\section{References}

[1] Auriault J.L. and Sanchez-Palencia E. Study of macroscopic behavior of a deformable porous medium. J. Méca. 1977; 16 (4):575-603.

[2] Sanchez-Palencia E. Nonhomogeneous Media and Vibration Theory, Lecture Notes in Physics, Springer, Berlin. 1980; 127.

[3] Levy T. Fluid flow through an array of fixed particles. Int. J. Engng. Sci. 1983; 21(1):11-23. 
[4] Whitaker S. Flow in porous media I: A theoretical derivation of Darcy's law. Transport in Porous Media. 1986; 1(1):3-25.

[5] Allaire G. Homogenization of the Stokes flow in a connected porous medium. Asymptotic Analysis. 1989; 2 (3):203-222.

[6] Sangani A. S. and Acrivos A. Slow flow past periodic arrays of cylinders with application to heat transfer. Int. J. Multiphase Flow. 1982, 8(3):193-206.

[7] Sangani A. S., Acrivos A. Slow flow through a periodic array of spheres. Int. J. Multiphase Flow. 1982; 3(4):343-360.

[8] Wang C.Y. Stokes flow through an array of rectangular fibers. Int. J. Multiphase Flow. 1996, 22(1):185-194.

[9] Wang C.Y. Stokes flow through a rectangular array of circular cylinders. Fluid. Dyn. Res. 2001, 29:65-80.

[10] Barrere J., Caltagirone J.P. and Gipouloux O. Détermination numérique de la perméabilité en milieu poreux périodique tridimensionnel. CR Acad. Sci. Paris, Sér. II. 1990; 310:347-352.

[11] Cioranescu D., Donato P. and Ene H.I. Homogenization of the Stokes problem with non-homogeneous slip boundary conditions. Math. Meth. Appl. Sc. 1996; 19(11):857-881.

[12] Alcocer F.J., Kumar V. and Singh P. Permeability of periodic porous media. Phys. Rev. E. 1999; 59 (1):711-714.

[13] Alcocer F. J. and Singh P. Permeability of periodic arrays of cylinders for viscoelastic flows. Phys. Fluids. 2002; 14 (7):2578-2581.

[14] Sawicki E. Numerical investigation of the fluid flow through rotating porous media at both the microscopic scale and macroscopic scale. Thèse, Université Joseph Fourier, Grenoble, 2004.

[15] Bernard D., Nielsen O., Salvo L. and Cloetens P. Permeability assessment by 3D interdendritic flow simulations on microtomography mappings of AlÜCu alloys. Mater. Sc. Engrg. A. 2005; 392:112-120.

[16] Stylianopoulos T., Yeckel A., Derby J.J., Luo X.J., Shephard M.S., Sander E.A. and Barocas V.H. Permeability calculation in three dimensional isotropic and oriented fiber network. Phys. fluids 2008; 20(12):123601.

[17] Malinouskaya I., Mourzenko V.V., Thovert J.-F. and Adler P.M. Wave propagation through saturated porous media. Phys. Rev. E 2008; 77:066302.

[18] Tahir M.A. and Tafreshi H.V. Influence of fiber orientation on the transverse permeability of fibrous media. Phys. Fluid. 2009, 21(8):08360.

[19] Koivu V., Decain M., Geindreau C., Mattila K., Bloch J.F. and Kataja M. Transport properties of heterogeneous materials. Combining computerised Xray micro-tomography and direct numerical simulations. Int. J. Comput. Fluid Dyn. 2009; 23(10):713-721. 
[20] Spaid M.A.A. and Phelan Jr. F.R. Lattice Boltzmann methods for modeling microscale flow in fibrous porous media. Phys. Fluids 1997; 9:2468.

[21] Koponen A., Kandhai D., Hellén E., Alava M., Hoekstra A., Kataja M., Niskanen K., Sloot P., and J. Timonen. Permeability of Three-Dimensional Random Fiber Webs. Phys. Rev. Lett. 1998; 80:716-719.

[22] Borne L. Harmonic Stokes flow through periodic porous media: a 3D Boundary Element Method. J. Comp. Phys. 1992; 99:214-232.

[23] Fortin M. Old and new finite element for icompressible flows. Int. J. Num. Meth. Fluids. 1981; 1:347-364.

[24] Arnold D.N., Brezzi F. and Fortin M. A stable finite element for the Stokes equations. Calcolo. 1984; 21(4):337-344.

[25] Arbogast T. and Brunson D.S. A computatonal method for approximating a Darcy-Stokes system governing a vuggy porous medium. Comput. Geosciences. 2007, 11(3):207-218.

[26] Monchiet V., Bonnet G. and Lauriat G. A FFT-based method to compute the permeability induced by a Stokes slip flow through a porous medium. C.R. Méca. 2009; 337(4):192-197.

[27] Moulinec H. and Suquet P. A fast numerical method for computing the linear and nonlinear mechanical properties of composites. C. R. Acad. Sci. 1994; 318(11):1417-1423.

[28] Michel J. C., Moulinec H. and Suquet P. Effective properties of composite materials with periodic microstructure: a computational approach. Comput. Methods. Appl. Mech. Engrg. 1999; 172:109-143.

[29] Le Droumaguet B., Lacombe R., Ly H.B., Guerrouache M., Carbonnier B. and Grande D. Engineering functional doubly porous PHEMA-based materials. Polymer, 2014; 55:373-379.

[30] Lee S.H., Shin H. Adv. Drug Delivery Rev. 2007, Vol. 59, pp. 339.

[31] Liu X.H., Ma P.X. Ann. Biomed. Eng. 2004, Vol. 32, pp. 477.

[32] Hoang M.T., Perrot C. Solid films and transports in cellular foams. J. Appl. Physics, 2012; 112(5):054911-6.

[33] Hoang M.T., Perrot C. Identifying local characteristic lengths governing sound wave properties in solid foams. J. Appl. Physics. American Institute of Physics (AIP), 2013; 113(8):084905-7.

[34] Nguyen T.-K., Monchiet V., Bonnet G. A Fourier based numerical method for computing the dynamic permeability of porous media. Eur. J. Mech. B/fluids. 2013; 37:90-98.

[35] Moulinec H. and Suquet P. A numerical method for computing the overall response of nonlinear composites with complex microstructure. Comput. Methods. Appl. Mech. Engrg. 1998; 157:69-94. 
[36] Monchiet V. and Bonnet G. A polarization based FFT iterative scheme for computing the effective properties of elastic composites with arbitrary contrast. Int. J. Num. Meth. Engrg. 2012; 89(11) 1419-1436.

[37] Sparrow E.M. and Loeffler A.L. Longitudinal laminar flow between cylinders arranged in a regular array. A.I.Ch.E.J. 1959, 5:325-330.

[38] Banerjee S., Hadaller G.I. Longitudinal laminar flow between cylinders arranged in a triangular array by a variational technique. J. Appl. Mech. 1973; 40:11361138.

[39] Drummond J.E. and Tahir M.I. Laminar viscous flow through arrays of parallel solid cylinders. Int. J. Multiphase Flow. 1984; 10:515-540.

[40] Larson R.E. and Higdon J.J.L. Microscopic flow near the surface of twodimensional porous media, Part I: axial flow. J. Fluid Mech. 1986; 166:449-472.

[41] Larson R.E. and Higdon J.J.L. Microscopic flow near the surface of twodimensional porous media, Part II: transverse flow. J. Fluid Mech. 1987; 178:119-136.

[42] Idris Z. and Orgeas L., Geindreau C., Bloch J.-F., Auriault J.-L. Microstructural effects on the flow law of power law fluids through fibrous media, Mod. Simul. Mater. Sc. Engrg. 2004; 12:995-1015.

[43] Chapman A.M. and Higdon J.J.L. Oscillatory Stokes flow in periodic porous media. Phys. Fluids A. 1992; 4 (10):2099-2116.

[44] Kadaksham A., Pillapakkam S.B. and Singh P. Permeability of periodic arrays of spheres. Mech. Res. Commun.. 2005; 32(6):659-665.

[45] Berdichevsky A.L. Cai Z. Perform permeability prediction by self consistent method and finite element simulations. Polymer composite, 1993, 14:132-143.

[46] Boutin C. Study of permeability by periodic and self consistent homogenization. Eur. J. Mech. A/solids. 2000, 19: 603-632. 